\title{
Genipin Crosslinked Blended Collagen-Chondroitin: A Promising Biomaterial Scaffold Candidate for Cartilage Reconstruction
}

\author{
Trimartani', Normalina Sandora ${ }^{2}$, Bambang Hermani' ${ }^{1}$, Jeanne A Pawitan ${ }^{3}$, Raden Ayu Anatriera ${ }^{1 *}$ \\ ${ }^{1}$ Department of Otorhinolaryngology-Head and Neck Surgery, Faculty of Medicine, University of Indonesia/Cipto Mangunkusumo \\ Hospital, Jakarta, Indonesia \\ ${ }^{2}$ Indonesia Medical Education and Research Institute, Faculty of Medicine, University of Indonesia, Jakarta, Indonesia \\ ${ }^{3}$ Department of Histology, Faculty of Medicine, University of Indonesia/Cipto Mangunkusumo Hospital, Jakarta, Indonesia
}

\section{ARTICLE INFO}

Article history:

Received March 16, 2021

Received in revised form September 10, 2021

Accepted October 28, 2021

\section{KEYWORDS:}

Biomaterial,

cartilage,

collagen-chondroitin,

genipin,

scaffold

\begin{abstract}
Tissue engineering offers a solution to the shortage of materials for cartilage reconstruction surgery by providing various potential biomaterial scaffolds. Tissue engineering utilizes biological or synthetic biomaterials as a scaffold for the host cells to repopulate and regenerate the tissue. The natural biomaterials such collagen and chondroitin imitates native cartilage matrix composition. Genipin as one of natural crosslinkers was added to improve the matrix biomechanical properties. This study was done to investigate biocomposition of blended collagen type 1 , collagen type 2, chondroitin sulphate (Col1-Col2-CS) and genipin for its cytotoxicity using human umbilical mesenchymal stem cells (hUCMSCs), surface morphology, and biochemical composition. Genipin-crosslinked collagen-chondroitin biocomposite showed a homogeneous shape while uncrosslinked biocomposite had rough surface and fibrillar folds size. Spectroscopy demonstrated both biocomposites had similar peak resemble to no alternation of the biocomposition by crosslinking. Both types of biocomposites were biocompatible and had no toxic effects, as compared to the cell colony only ( $p$ value $=\mathbf{0 . 2 6}$ ). The conclusion are blended composite of collagen chondroitin crosslinked with genipin had generated a fine microstructure scaffold with smaller pore size, had similar biomolecular component spectrum absorption, and no exhibition of residual toxicity.
\end{abstract}

\section{Introduction}

Tissue engineering have great potential in cartilage defects in any reconstructive surgery, focusing cartilage as one of most important aspects of structural function, for example in otorhinolaryngology and facial surgery. Depending on the tissue defect, conventional treatments use autograft or allograft cartilage for reconstruction. This approach has been shown to increase patient's quality of life along, however several limitations exist. The uses of autologous cartilage taken from the patients' body is limited by the donor site supply and may cause secondary morbidity, increased risk of complications, and increased cost. The uses of allogeneic source, on the other hand, are constrained by possible rejection and infection transmissions. Tissue engineering comes as alternative strategy to overcome problems associated with autograft

\footnotetext{
* Corresponding Author

E-mail Address: anatriera@gmail.com
}

and allograft approach (Cao et al. 2014; Chen et al. 2016). The natural biomaterials might be excellent candidate for cartilage reconstruction. Natural biomaterial has cellular adhesion molecules; hence they are recognized by the host cells, enabling them to infiltrate and proliferate into the graft when it is implanted. Collagen type 1 (Col1), collagen type 2 (Col2), and chondroitin sulphate (CS) had been extensively studied as the scaffold for chondrocytes as they are biocompatible. Nonetheless, they showed no inherent rigidity and stiffness (Chen et al. 2016; Portalation et al. 2016). Mechanical property of scaffold is important to derive cellular differentiation and growth. One of the most favored combination scaffold to improve stiffness is by adding crosslinking agent. However, genipin as crosslinking agent is derived from natural compound, extracted from fruit of Gardenia jasminoides plant which more subtle and stable (Nagaoka et al. 2014; Shah et al. 2019). In this study, genipin was used as crosslinker to collagenchondroitin biocomposite. 
Both collagen and chondroitin was studied predominantly fascinating because it imitates closest to native cartilage matrix composition (Urbanski 2015). There were no specific previous studies that demonstrated effect of genipin into blended two types of collagen along with chondroitin added. This investigation was aimed to develop a cartilage biomaterial scaffold that provide appropriate microenvironment for deriving cells into chondrocytes, and can be a candidate for studying cartilage replacement in the future.

\section{Materials and Methods}

\subsection{Scaffold Preparation}

Collagen type 1 (bovine tendon, Sigma-Aldrich, UK) and collagen type 2 (bovine nasal septum, SigmaAldrich, UK) was solubilized in $0.01 \mathrm{mM}$ acetic acid to make $10 \mathrm{mg} / \mathrm{ml}$, and $5 \mathrm{mg} / \mathrm{ml}$ solution, respectively. The mixture was incubated at $45^{\circ} \mathrm{C}$ for 30 minutes, then the $\mathrm{pH}$ was adjusted up to 7 . Chondroitin sulphate (shark cartilage, Sigma-Aldrich, UK) was prepared at $30 \mathrm{mg} / \mathrm{ml}$, dissolved in $130 \mathrm{mM} \mathrm{NaCl}$, then added into the collagen solution (ratio 1:3) as modified from other study (Ashokkumar et al. 2012; Barco et al. 2018). The hydrogel (Col1-Col2-CS) was then split into two groups, crosslinked with genipin (Sigma-Aldrich, UK), and without (each $n=3$ ). The genipin was optimized by the researcher at $1 \mathrm{mM}$ concentration. Both groups were incubated at $37^{\circ} \mathrm{C}$ for 72 hours. The composite hydrogels of both groups were then lyophilized for 24 hours.

\subsection{Contact and Extract Cytotoxicity}

In this study, stem cells used in cytotoxicity test were derived from hUCMSCs. The MSCs characteristics was identified using flowcytometry, using CD73 (99\%, Biolegend, USA), CD90 (99\% Biolegend, USA), CD105 ( $80 \%$, Biolegend, USA), adhered to the plastics and had fibroblastic shape in standard culture conditions (Dominici et al. 2006).

\subsubsection{Contact Cytotoxicity}

All composite samples $(50 \mu \mathrm{l})$ of both groups were placed in each well of a six-well plate. Cell suspension of the hUCMSCs was prepared at $100,000 \mathrm{cell} / \mathrm{ml}$ in Dulbecco's Modified Eagle Medium (DMEM, SigmaAldrich, UK) supplement with $10 \%$ (v/v) Fetal Bovine Serum (FBS), Penicillin/Streptomycin $100 \mathrm{U}, 2 \mathrm{mM}$ L-glutamine, and left incubated for 48 hours at $37^{\circ} \mathrm{C}$, $5 \%(\mathrm{v} / \mathrm{v}) \mathrm{CO}_{2}$ in air. The samples were imaged using Brightfield microscope (Axio, V2, Zeiss).

\subsubsection{Extract Cytotoxicity}

All samples (300 mg) of both groups were incubated in the $3 \mathrm{ml}$ of DMEM only, for 72 hours, $240 \mathrm{rpm}$, at $37^{\circ} \mathrm{C}$. The extracted medium was used to replace the culture medium in each well of the 96-well plate after incubation for two days seeded with 30,000 cells hUCMSCs per well $(n=3)$. After 48 hours of incubation with the extracted medium, at $37^{\circ} \mathrm{C}, 5 \%(\mathrm{v} / \mathrm{v}) \mathrm{CO}_{2}$, the cells were lysed and incubated with double strength medium culture $20 \%(\mathrm{v} / \mathrm{v}$ ) FBS. The ATPLite-M reagent (Perkin Elmer, US) was added to each plate and isolated from any source of light (Elmer 2013). Measurement of luminescence using VarioSkan Lux (Thermo, Germany) was performed and the data were collected using SkanIt software (Thermo, Germany).

\subsection{Surface Morphology}

All samples $(n=3)$ were lyophilized and prepared for scanning electron microscope (SEM; Inspect F50, FEI, United States). Samples were critical point dried using stub and vacuum coater, sputtered with thin gold film (Emitech, K550) for 90 seconds and studied under SEM Inspect F50®.

\subsection{Fourier-Transform Infrared Spectroscopy (FTIR)}

Both composite hydrogels were analysed using FTIR spectroscopy at the mid infrared (MIR) spectral range (4000-400 $\left.\mathrm{cm}^{-1}\right)$, measured using OPUS®.

\subsection{Alcian Blue Staining}

The hUCMSCs was suspended at $10^{7}$ cells $/ \mathrm{ml}$ in the composite hydrogel with and without genipin, incubated as $10 \mu \mathrm{lmicro-drops}$ for three days, cultured using standard culture medium (DMEM added with Penicillin/Streptomycin 100U, 2 mM L-glutamine, and $10 \%(v / v)$ FBS). The colony was stained using Alcian Blue (SkyTec, USA). Briefly, the colony was washed by PBS pH 7.4, fixed using neutral buffer formaldehyde 10\% (v/v) for 15 min, stained with Alcian Blue for 15 minutes after PBS washes. The cells were imaged using Brightfield microscope (Axio, V2, Zeiss).

\subsection{Statistical Analysis}

Data was analysed using IBM SPSS software v.20 (IBMCoorporation, Armonk,NY,USA).The parameter's differences were conducted using one-way ANOVA, and the multiple comparison was corrected using Sidak test. Error bars denote the mean $(n=3) \pm 95 \%$ $\mathrm{CI}$, p value $<0.05$ indicates significant. 


\section{Results}

The crosslinked group were shown to form a solid structure, were able to retain matrix shape and had generated the macroscopically visible pores. The crosslinked hydrogel had turned dark blue as the sign of crosslinked process. The crosslinked Col1-Col2-CS hydrogel at Figure 1 showed a homogeneous surface morphology, had smaller pore size as compared to the uncrosslinked Col1-Col2-CS hydrogel which had large fibrillar folds.

Biomolecular profile was analyzed using FTIR to investigate the material microstructure indirectly. It was demonstrated that the biomolecular component of the biocomposites Col1-Col2-CS hydrogel; crosslinked and un-crosslinked had similar peak resemble no alternation of the biocomposition by crosslinking. Spectrum absorption peaks were identified at 3396 $\mathrm{cm}^{-1}, 2193 \mathrm{~cm}^{-1}, 1650 \mathrm{~cm}^{-1}, 1565 \mathrm{~cm}^{-1}, 1413 \mathrm{~cm}^{-1}, 1344$ $\mathrm{cm}^{-1}, 1242 \mathrm{~cm}^{-1}$, and $1019 \mathrm{~cm}^{-1}$. The absorbent at 1820 to $1600 \mathrm{~cm}^{-1}$ belonged to carbonyl group $(\mathrm{C}=0), 3400$ $2400 \mathrm{~cm}^{-1}$ for carboxylic acid group $(-\mathrm{OH}), 1350 \mathrm{~cm}^{-1}$ to $1470 \mathrm{~cm}^{-1}$ indicates the alkane group $(\mathrm{C}-\mathrm{H})$, while the area of 1180 to $1360 \mathrm{~cm}^{-1}$ for amine group (C-N). Similar absorption results indicated that genipin does not damage the structure of the scaffold molecule itself as seen in Figure 2.
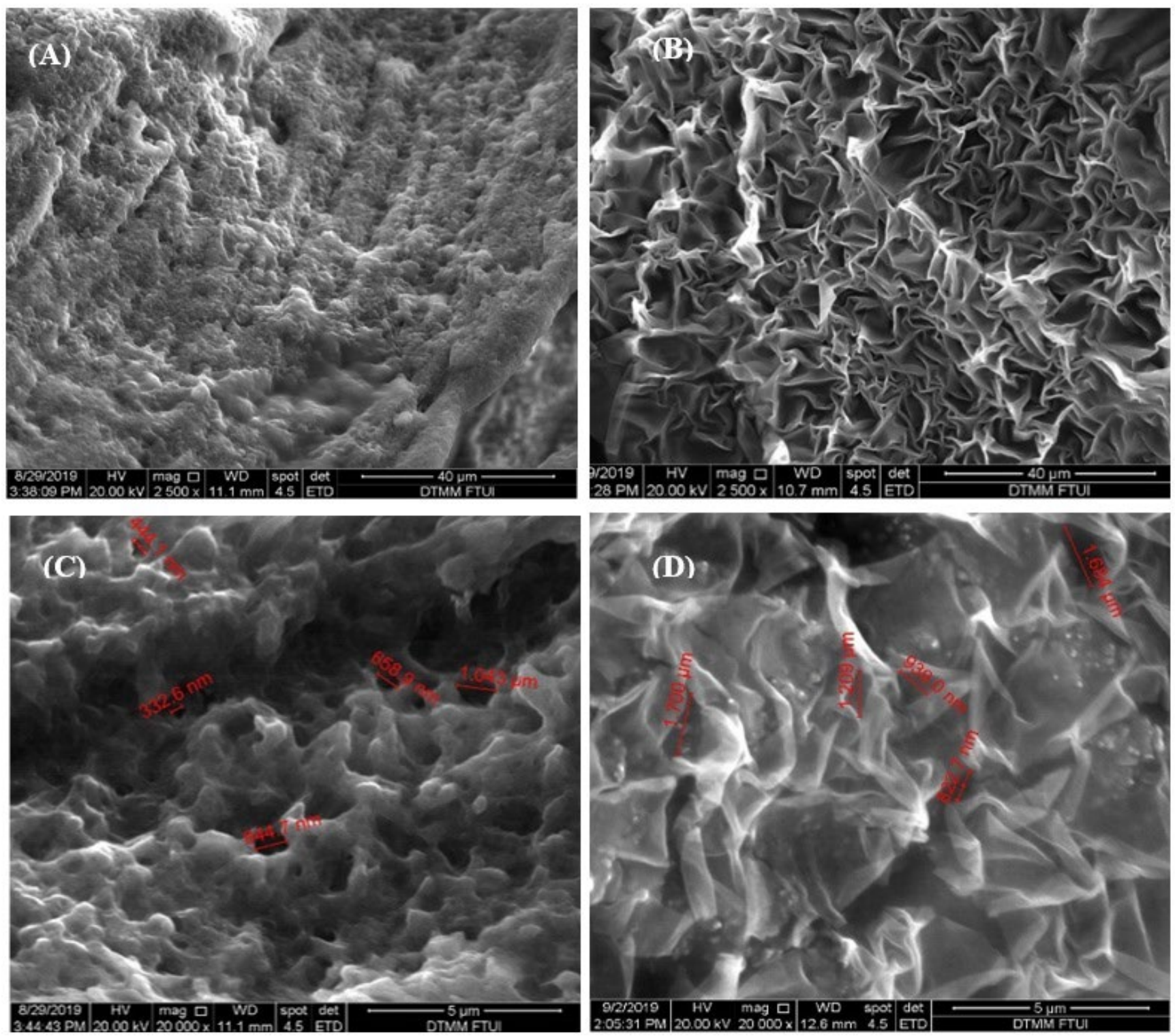

Figure 1. Scanning electron microscope images of $(A, C)$ the crosslinked, and $(B, D)$ the un-crosslinked Col1-Col 2-CS hydrogel. The (A, B) images were captured using SEM at 2500x magnification, while the (C, D) were at 20000x magnification 
The contact cytotoxicity in Figure 3 showed that the hUCMSCs colony able to occupy the matrices of the composite hydrogel crosslinked using $1 \mathrm{mM}$ genipin, which was not different as compared to the composite hydrogel using uncrosslinked matrix. The cell morphology incubated with both the matrices showed no different to the colony of cells without matrices. These indicate that both matrix types did not disturb the cellular metabolism, nor the proliferation. These were seen in the extract toxicity in Figure 4, where the ATP quantified from the cell colony after incubation with the matrix extract showed no difference to the cell colony only $(\mathrm{p}=0.26)$.

In this study, there were no different of the ATP level between the cell colony only to the biocomposite with and without genipin. This indicate that the addition of genipin to the biocomposite did not impact its toxicity ( $\mathrm{p}$ value $=0.26$ )

The alcian blue staining showed that the crosslinked Col1-Col2-CS hydrogel had retained GAGs, while the un-crosslinked and the cell only showed no staining retained. The hUCMSCs had grown into the hydrogel no different in both groups, indicated that the genipin might not toxic to the cells. The culture of hUCMSCs in the Col1-Col2-CS hydrogels showed that the matrix were degraded in a high speed for three days, nearly half of the matrix of the crosslinked group were gone, while the un-crosslinked matrix was degraded faster (Figure 5).

\section{Discussion}

Studies in search for an ideal biomaterial, the synthetics or natural, as cartilage replacement is extensive as its significant needs in the field of musculoskeletal, degenerative diseases, and in otolaryngology reconstruction. Mesenchymal stem cells have ability to differentiate into tissue-specific cells and already used in most tissue engineering studies (Meiliana et al. 2016). Mesenchymal stem cells (MSC) implanted in chondroitin-collagen scaffolds showed higher immunosuppression activity as compared to those implanted in collagen onlybased scaffolds. This evidence was demonstrated by significantincrease in nitric oxide $(\mathrm{p}<0.01)$ subsequent to stimulation with TNF- $\alpha$ on mesenchymal stem cells implanted in collagen-chondroitin scaffolds (Corradetti et al. 2016).

Genipin, besides a crosslinker, were also shown to improve cellular proliferation and reduce cellular

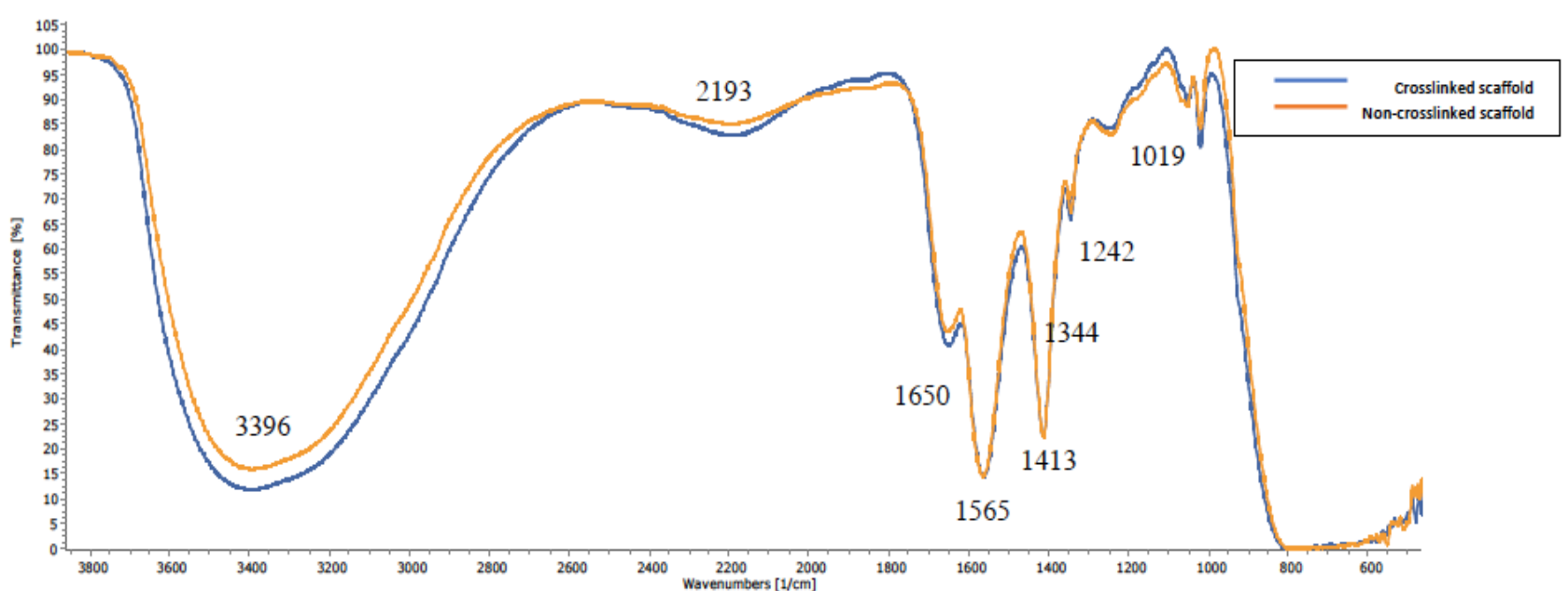

Figure 2. FTIR results in both Col1-Col2-CS hydrogel with and without crosslinking. Spectrum absorption peaks were identified at $3396 \mathrm{~cm}^{-1}, 2193 \mathrm{~cm}^{-1}, 1650 \mathrm{~cm}^{-1}, 1565 \mathrm{~cm}^{-1}, 1413 \mathrm{~cm}^{-1}, 1344 \mathrm{~cm}^{-1}, 1242 \mathrm{~cm}^{-1}$, and $1019 \mathrm{~cm}^{-1}$ 

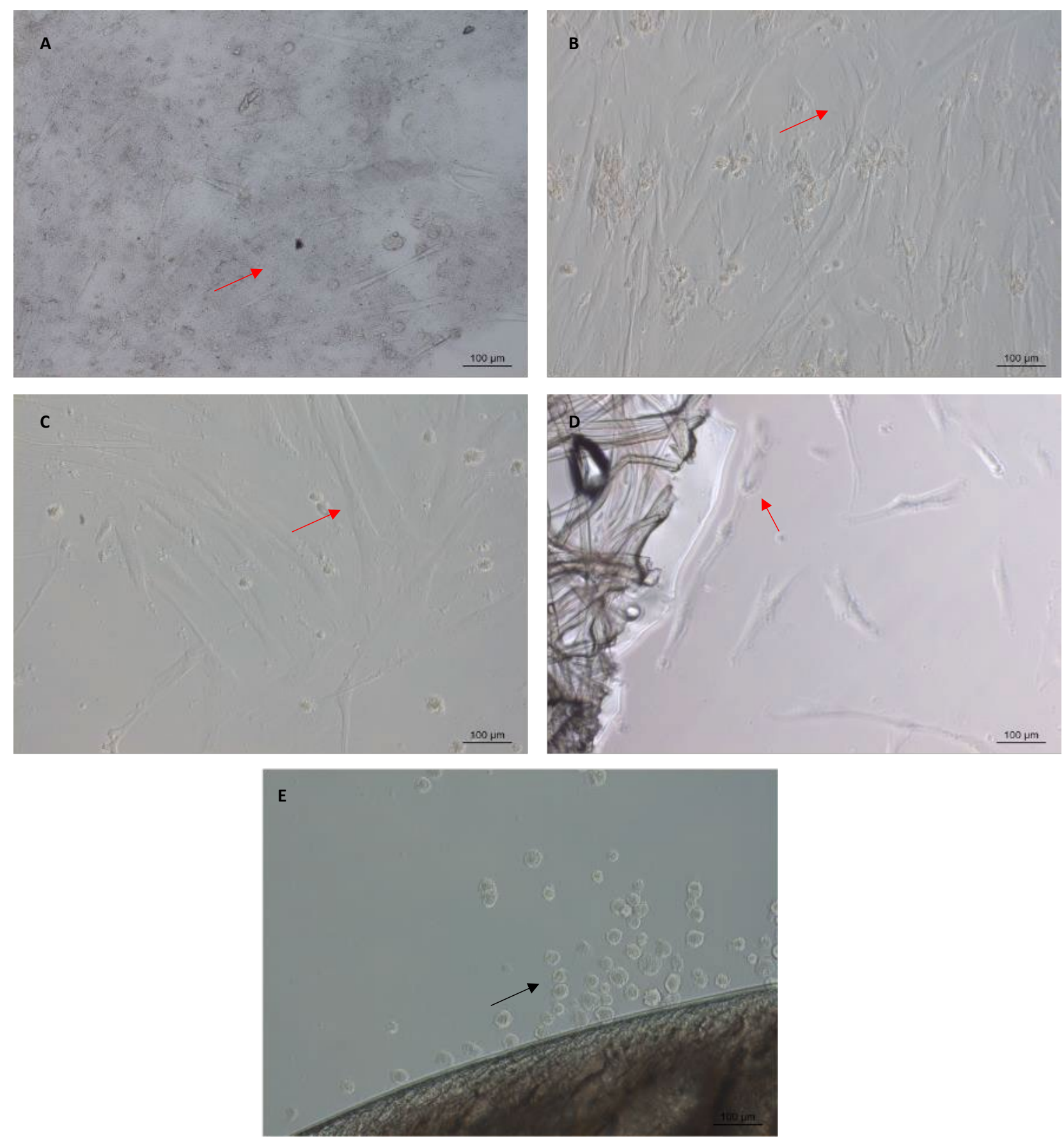

Figure 3. Contact cytotoxicity, (A) crosslinked with genipin, (B) un-crosslinked, (C) cell only, (D) negative control (Steristrip), (E) positive control (cyanoacrilic). Images were visualised using Brightfield microscope (AxioV2, Zeiss), scale bar denotes 100x magnification. The red arrow pointed to the viable hUCMSC, while the black arrow to non-viable hUCMSC 


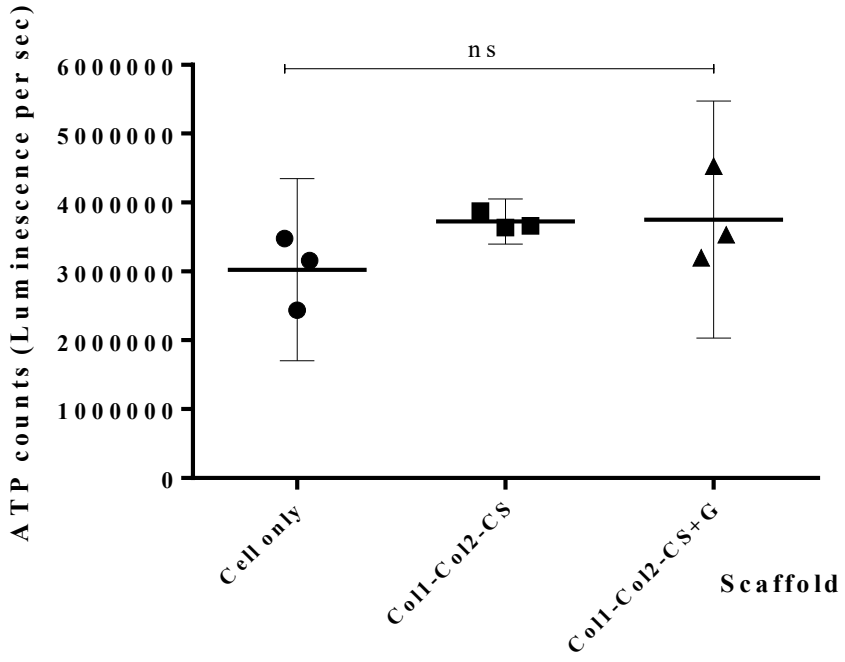

Figure 4. Extract cytotoxicity, using ATP quantification of Col1-Col2-CS hydrogel crosslinked with genipin and without, compared to the cell only. The cell only group was used as the control. Data was analysed using one-way ANOVA, and the multiple comparison was corrected using Sidak test. Error bars denote to the mean $(n=3) \pm 95 \% C I$, $p$ value $<0.05$ indicates significant. the $\mathrm{p}$ value $=0.256$
degradation(Madhavan et al.2011; Wang and Tsai 2013). Genipin performed as better crosslinker improves the elasticity and stability collagen scaffolds as mentioned on the study (Wang and Tsai 2013). Genipin crosslinked Col1-Col2-CS biocomposites showed more homogeneous surface morphology, fine pore size as compared to the uncrosslinked in this study, respectively. It is shown that adding such genipin concentration can affect the pore size (Bi et al. 2011). The reason for this observation can be related to collagen scaffold would be crosslinked and rehydrated concurrently while being submersed in genipin. Then, it would lead to pore structure collapse and fusions of the collagen fibers. Crosslinking process also enhances the scaffold structural stability and allowing more water retention ability (Mekhail et al. 2011; Shah et al. 2019).

The genipin-crosslinked composite hydrogel in this study showed similar absorbent spectrum to those without genipin group. Similar spectra peaks were observed at some absorption area. The only different peak seen at $3750-3700 \mathrm{~cm}^{-1}$ found in the Col1-Col2CS hydrogel without genipin. This finding is align to the study of Urbanski who also observed the presence

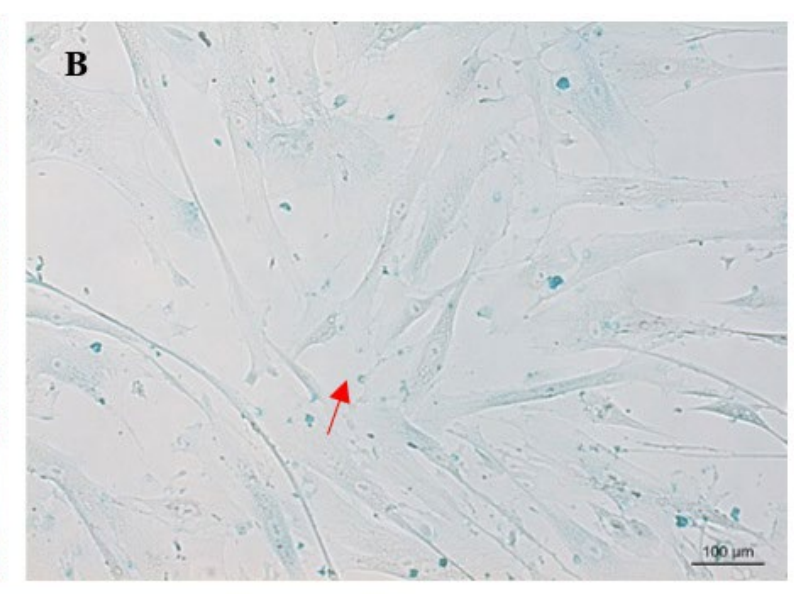

C
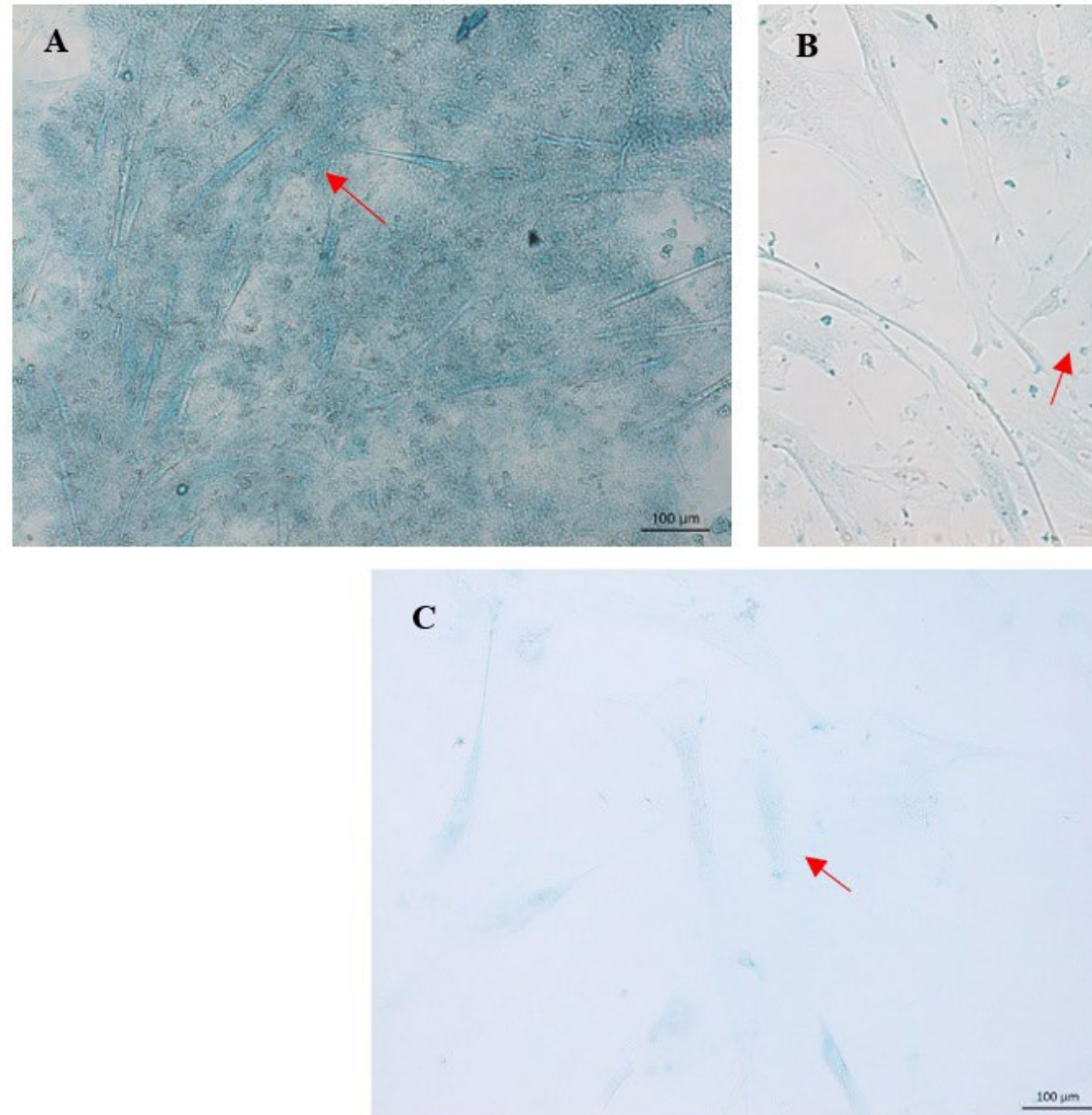

Figure 5. Alcian blue staining of the Col1-Col2-CS hydrogel, (A) crosslinked with genipin, (B) un-crosslinked, (C) cell only. Images were visualised using Brightfield microscope (AxioV2, Zeiss), scale bar denotes 100x magnification. Red arrows pointed to the viable hUCMSC 
of several wave peaks in the absorption area of 3750$3600 \mathrm{~cm}^{-1}$ (Urbanski 2015). This wave peak is generated by the activity of the hydroxyl group $(-\mathrm{OH})$ and free carboxyl group $(-\mathrm{COOH})$, in which the $\mathrm{O}-\mathrm{H}$ chain are twisted. Due to crosslinking process, this $\mathrm{O}-\mathrm{H}$ chain had bound to other molecules (not in a free state), therefore the Col1-Col2-CS hydrogel undergone crosslinking process exhibited no peak at this $3750-3600 \mathrm{~cm}^{-1}$. To conclude, apparently genipin crosslinking to the Col1-Col2-CS hydrogel had not changing the molecular composition of the hydrogel, albeit that the collagen fibers were crosslinked, the molecular structure were still maintained (Pavia et al. 2015; Barco et al. 2018).

This study also found that both groups were biocompatible, meaning that this biocomposite were not toxic. The extract cytotoxicity to determine cellular viability, by counting the ATP contain of the cells. ATP assay is the most rapid and most sensitive cellular viability assay (Loh and Choong 2013; Klejin et al. 2016). The ATP assay counts the beam of the luminescence light generated by the reaction of ATP to luciferase and d-luciferin added to the sample (Elmer 2013). Current study showed that the ATP count in the hUCMSCs colony incubated with the Col1-Col2-CS hydrogel crosslinked with genipin were not different as compared to the colony incubated with the Col1-Col2-CS hydrogel without genipin, and as compared to the cell colony only. Cells in a three-dimensional culture are more viable and able to survive, perhaps due to the interconnected pores in a 3D structure allow nutrient transport, cell proliferation and tissue formation. The 3D structure supports nutrient, oxygen, metabolites exchange, allow spaces for various biological factors such as growth factors, exosomes, for cell to cell communication (Loh and Choong 2013).

\section{References}

Ashokkumar, M., Sumukh, K.M., Murali, R., Narayanan, N.T., Ajayan, P.M., Thanikaivelan, P., 2012. Collagenchitosan biocomposites produced using nanocarbons derived from goatskin waste. Carbon. 50, 5574-5582. https://doi.org/10.1016/j.carbon.2012.08.006

Barco, A., Ingham, E., Fisher, J., Fermor, H., Davies, R.P.W., 2018. On the design and efficacy assessment of self-assembling peptide-based hydrogelglycosaminoglycan mixtures for potential repair of early stage cartilage degeneration. J. Pep. Sci. e3114. https://doi.org/10.1002/psc.3114

Bi, L., Cao, Z., Hu ,Y., Song, Y., Yu, L., Yang, B., Mu, J., et al. 2010. Effects of different cross-linking conditions on the properties of genipin-cross-linked chitosan/ collagen scaffolds for cartilage tissue engineering. J. Mater. Sci. Mater. Med. 22, 51-62. https://doi.org/10.1007/ s10856-010-4177-3

Cao, Z., Dou, C., Dong, S., 2014. Scaffolding biomaterials for cartilage regeneration. J. Nanomater. 2014, 1-8. https://doi.org/10.1155/2014/489128
Chen, F., Liu, X., 2016. Advancing biomaterials of human origin for tissue engineering. Prog. Polym. Sci. 53, 186-168. https://doi.org/10.1016/j.progpolymsci.2015.02.004

Corradetti, B., Taraballi, F., Minardi, S., Eps J Van, Cabrera, F., W.Francis L., et al. 2016. Chondroitin sulfate immobilized on a biomimetic scaffold modulates inflammation while driving chondrogenesis. Stem. Cells. Transl. Med. 5, 670-682.https://doi.org/10.5966/ sctm.2015-0233

Dominici, M., Le Blanc, K., Mueller, I., Slaper-Cortenbach, I., Marini, F.C., Krause, D.S., Deans, R.J., Keating, A., Prockop, D.J., Horwitz, E.M., 2006. Minimal criteria for defining multipotent mesenchymal stromal cells. The International Society for Cellular Therapy position statement. Cythotherapy. 8, 315-317. https://doi. org/10.1080/14653240600855905

Elmer, P. 2013. ATPlite Luminescence ATP detection assay system. PerkinElmer Ltd, Waltham.

Kleijn, A., Kloezeman, J.J., Balvers, R.K., Kaaij M Van Der, Dirven, C.M.F., Leenstra, S., et al. 2016. A systematic comparison identifies an ATP-based viability assay as most suitable read-out for drug screening in glioma stem-like cells. Stem. Cells. Int. 2016, 5623235. https:// doi.org/10.1155/2016/5623235

Loh, Q.L., Choong, C., 2013. Three-dimensional scaffolds for tissue engineering applications: role of porosity and pore size. Tissue Eng Part B Rev. 19, 485-502. https:// doi.org/10.1089/ten.teb.2012.0437

Madhavan, K., Belchenko, D., Tan, W., 2011. Roles of genipin crosslinking and biomolecule conditioning in collagen-based biopolymer: potential for vascular media regeneration. J. Biomed. Mater. Res. 97, 16-26. https://doi.org/10.1002/jbm.a.33006

Meiliana, A., Mustika, Dewi, N., Wijaya, A., 2016. Stem cell therapy in wound healing and tissue regeneration. Indones. Biomed.J. 8, 61-70. https://doi.org/10.18585/ inabj.v8i2.191

Mekhail, M., Wong, K., Padavan, D., Wu, Y., Gorman, D., Wan, W., 2011. Genipin-cross-linked electrospun collagen fibers. J. Biomater. 22, 2241-2259. https:// doi.org/10.1163/092050610X538209

Nagaoka, H., Walter, R., Boushell, L.W., Miguez, P.A., Burton, A., Ritter, A., Yamauchi, M., 2014. Characterization of genipin-modified dentin collagen. Biomed Res. Int. 2014,1-7. https://doi.org/10.1163/092050610X538209

Pavia, D.L., Lampman, G.M., Kriz, G.S., Vyvyan, J., 2015. Introduction to Spectroscopy. fifth ed. Cengage Learning, Washington.

Portalation, N.V., Kilmer, C.E., Panitch, A.L.J., 2016. Characterization of collagen type I and II blended hydrogels fot articular cartilage tissue engineering. Biomacromolecules. 17, 3145-3152. https://doi. org/10.1021/acs.biomac.6b00684

Shah, R., Stodulka, P., Skopalova, K., Saha, P., 2019. Dual crosslinked collagen/chitosan film for potential biomedical applications. Polymers. 11, 2094. https:// doi.org/10.3390/polym 11122094

Urbanski, L., 2015. Fabrication and characterization of collagen, hyaluronic acid and chondroitin sulfate scaffolds for cartilage tissue engineering applications [Thesis]. Tampere: Tampere University of Technology.

Wang, P., Tsai, W., 2013. Modulation of the proliferation and matrix synthesis of chondrocytes by dynamic compression on genipin-crosslinked chitosan/ collagen scaffolds. J. Biomater. Sci. 24, 507-519. https://doi.org/10.1080/09205063.2012.696310 